\title{
Lutter contre le paludisme en réduisant sa transmission? Présentation de la controverse
}

\author{
Vincent Robert, Jean-François Trape
}

La place de la lutte antivectorielle dans la lutte antipaludique en zone de forte ou moyenne endémie palustre (soit essentiellement en Afrique tropicale rurale) est actuellement l'objet d'une controverse. Le suivi à court terme des interventions ayant réduit la transmission, par exemple par pulvérisation intradomiciliaire d'insecticides ou par utilisation de moustiquaires imprégnées de pyréthrinoïde, montre une réduction importante de la morbidité et de la mortalité palustres. A l'opposé, la comparaison de plusieurs situations où les niveaux de transmission different, suggère que, sur le long terme, l'abaissement du niveau de transmission n'a pas d'autre conséquence que de retarder l'âge moyen de survenue des accès palustres sans modifier l'importance globale de la morbidité et de la mortalité dues au paludisme. L'apparente contradiction entre les résultats observés reflète des approches différentes. Elle est avant tout révélatrice de l'importance des défis que soulève la lutte contre le paludisme. es relations entre l'intensité de la transmission des agents de maladies parasitaires et l'importance de la morbidité qui leur est associée constituent un domaine des interactions hôte/vecteur/parasite dont les implications sont majeures en santé publique. Dans certains cas, le schéma de ces relations est relativement simple. C'est, par exemple, le cas de la maladie du sommeil à Trypanosoma brucei qui est habituellement mortelle en absence de traitement pour les personnes ayant reçu une seule piqûre infectante de glossine. De même, ce schéma est relativement simple, quoique radicalement différent, dans le cas de l'onchocercose, type de la maladie d'accumulation, dont les conséquences sont directement proportionnelles au nombre reçu de piqûres de simulies infectées; la cécité est l'aboutissement d'une exposition de plusieurs années à de nombreux

de transmission peut varier considéravecteurs infectés. de transmission peut varier considéra-

\section{La transmission et ses conséquences}

Dans le cas du paludisme, l'immunité partielle qui est progressivement acquise par les individus fréquemment réinfectés amène à distinguer plusieurs types de situations épidémiologiques. On oppose classiquement les zones épidémiques aux zones d'endémie stable.

Dans les zones épidémiques, la transmission est, par définition, exceptionnelle. La population n'étant habituellement pas en contact avec le parasite, elle ne bénéficie d'aucune protection immunitaire. Chaque accès palustre provient d'une unique piqûre infectée d'anophèle et le risque de décès en l'absence de traitement est élevé. Cette situation est aussi celle des voyageurs provenant de pays indemnes de paludisme qui sont infectés à l'occasion d'un séjour en zone d'endémie.

Dans les zones endémiques, le niveau dans une moindre mesure, d'une année à l'autre. Ainsi, la transmission peut dépasser 1000 piqûres infectées d'anophèle par personne et par an dans certains villages d'Afrique, notamment en Afrique centrale [1]. D'une façon schématique, l'ordre de grandeur du niveau de la transmission, qui est liée au rythme saisonnier et à l'intensité des précipitations, est respectivement de l'ordre de 10,100 et 200 piqûres infectées par personne et par an dans les villages des régions du Sahel, de savane et de forêt en Afrique tropicale. En revanche, dans les autres régions du monde dont les espèces vectrices sont moins efficaces, la transmission est presque toujours inférieure à une piqûre infectée par personne et par an. Il est ainsi nécessaire de distinguer le cas des zones de forte et moyenne endémie (ou zones d'endémie stable, que l'on peut caractériser par une transmission toujours supérieure à une piqûre infectée par personne et par an) de celui des zones de faible endémie (ou zones d'endémie instable car la trans- 
mission, outre son faible niveau, présente généralement des variations interannuelles importantes).

En zone de forte transmission, chaque piqûre d'anophèle infecté n'entraîne pas un accès palustre. Cela est dû à l'acquisition progressive d'une immunité partielle qui se renforce naturellement tout au long de la vie avec la répétition des inoculations. L'essentiel de la morbidité et la quasi-totalité de la mortalité dues au paludisme dans les régions de forte endémie sont concentrés chez les jeunes enfants. Inversement, en zone de faible endémie, la situation est proche de celle des zones épidémiques, toutes les classes d'âge étant concernées par la maladie palustre et par le risque de décès. Toutefois, peu de données précises sont disponibles et les études associant un recueil de données entomologiques, parasitologiques, cliniques et démographiques sont lourdes à réaliser et encore peu nombreuses. De plus, ce n'est que récemment qu'ont été développées des méthodes de mesure de la morbidité et la mortalité palustres suffisamment précises pour permettre des études quantitatives par classes d'âge. Cela explique que l'analyse des relations entre le niveau de la transmission et l'importance de la pathologie palustre reste inachevée.

\section{L'amélioration de la situation palustre par la réduction de la transmission}

Dans les zones épidémiques ou de faible endémie, une relation proportionnelle directe existe entre la quantité de transmission, d'une part, la morbidité et la mortalité palustres, d'autre part. Dans ce contexte, toutes les classes d'âge bénéficient de la plus faible transmission possible. La réduction de la transmission par une lutte antivectorielle se traduit par une diminution de la morbidité et de la mortalité.

Dans les zones de moyenne et de forte endémie, les programmes de lutte les plus ambitieux envisagent seulement une réduction ou un arrêt temporaire de la transmission. Pour des raisons techniques et/ou économiques, le maintien de l'arrêt com- plet de la transmission (qui équivaudrait à une éradication du paludisme), ou son maintien à un très faible niveau, ne sont pas actuellement envisageables. Un débat est ouvert sur la question de savoir si une réduction partielle de la transmission sera suivie d'une réduction durable de la morbidité et de la mortalité.

\section{La controverse}

La controverse concerne uniquement les zones d'endémie moyenne et forte. Elle découle de l'apparente contradiction entre les résultats obtenus à partir des deux principales méthodes utilisées jusqu'à maintenant pour étudier les relations entre transmission, morbidité et mortalité: (1) le suivi d'interventions visant à réduire la transmission, essentiellement par la pulvérisation intradomiciliaire d'insecticide ou par l'utilisation de moustiquaires de lit imprégnées d'insecticide [2], (2) la comparaison de plusieurs situations dans lesquelles les niveaux de transmission diffèrent. Quelques exemples doivent ici être présentés.

\section{Le suivi d'une intervention réduisant la transmission}

-A Kisumu, région de très forte endémie au Kenya, un projet de l'OMS a consisté à effectuer pendant deux ans des pulvérisations intradomiciliaires d'insecticide dans une zone rurale dont la population totale était de 17000 personnes. Chez les enfants de moins d'un an, l'incidence parasitaire a été réduite de $96 \%$, la prévalence parasitaire de $67 \%$ et la mortalité générale de $41 \%$ par rapport à la zone témoin. De même, la mortalité générale pour toutes les tranches d'âge a été réduite de $43 \%$ [3].

- Dans des rizières de la Rusizi, zone de moyenne endémie au Burundi, pendant six ans (1985-1990), une pulvérisation annuelle intradomiciliaire d'un insecticide (associé à partir de la troisième année à un système de drainage des eaux pluviales et, pour $45 \%$ de la population, à la mise en place de moustiquaires imprégnées d'insecticide) a sup- primé le pic de transmission de paludisme de fin de la saison des pluies. La population protégée atteignait 80000 personnes en 1990. Le niveau initial de la transmission a été évalué à 77 piqûres d'anophèles infectés par personne et par an. La prévalence parasitaire chez les moins de 5 ans est passée de $70 \%$ à $8 \%$ en six ans. De même, dans les gouttes épaisses positives, les fortes charges parasitaires ont diminué de $35 \%$ à moins de $5 \%$ [4].

- Les moustiquaires imprégnées de pyréthrinoïde réduisent également grandement la transmission. Dans la rizière de la vallée du Kou, au Burkina Faso, l'imprégnation des moustiquaires des villageois a entraîné une diminution de $96 \%$ de la transmission pendant un an [5]. D'autres études ont retrouvé un taux de réduction similaire et ont montré que la densité parasitaire était nettement diminuée [6]. Une méta-analyse portant sur dix essais de moustiquaires imprégnées dans des zones de forte endémie a montré qu'après un an l'incidence de l'infection était réduite de $24 \%$ par rapport aux moustiquaires simples et de $50 \%$ par rapport à l'absence de moustiquaires [7]. Enfin, trois études récentes, en Gambie, au Kenya et au Ghana, ont montré que les moustiquaires imprégnées entraînaient après un ou deux ans d'utilisation une réduction de la mortalité générale des enfants comprise entre $17 \%$ et $33 \%[8,9,10]$.

\section{Comparaisons de plusieurs zones sans intervention}

- Une étude à Brazzaville, Congo, a montré que la transmission était extrêmement variable d'un quartier à l'autre, variant de moins d'une piqûre d'anophèle infecté par personne tous les trois ans à plus de 100 piqûres infectées par personne et par an. De même, la prévalence du paludisme chez les écoliers variait de $3 \%$ à $81 \%$. Malgré cela, la fréquence des accès palustres graves et des décès par paludisme était similaire dans tous les quartiers de la ville, la seule différence portant sur l'âge moyen des enfants présentant un paludisme grave (ces enfants étaient plus jeunes 
dans les quartiers à forte transmission). Dans le cas de Brazzaville, l'intensité de la transmission n'était donc pas un déterminant important de la fréquence des accès graves et de la mortalité palustre [11].

- La comparaison de la zone d'Ifakara, Tanzanie, où la transmission était de 300 piqûres d'anophèles infectés par personne et par an et de la zone de Kilifi, Kenya, où la transmission était 33 fois moindre, n'a pas montré de différence pour le taux d'accès graves chez les enfants de moins de cinq ans. En revanche, la fréquence relative des formes cliniques des accès graves était différente (les anémies graves et les atteintes neurologiques étant respectivement dominantes) et les nourrissons étaient deux fois plus souvent atteints à Ifakara qu'à Kilifi. Cela suggère qu'une éventuelle réduction de la transmission de $98 \%$ à Ifakara (qui permettrait d'atteindre un niveau comparable à celui de Kilifi) ne modifierait probablement pas le nombre d'accès palustres graves chez les enfants [12].

- La transmission du paludisme dans neuf sites côtiers du district de Kilifi a été évaluée, selon les sites, d'environ 1 à 60 piqûres d'anophèles infectés par personne et par an. Dans le même temps l'incidence annuelle des accès palustres graves chez les enfants de moins de 4 ans a été évaluée selon les sites de 9 à 38 pour mille enfants. Les différences d'incidence n'étaient pas liées au niveau de la transmission [13].

- Des études sur la mortalité palustre ont été conduites indépendamment en Afrique dans des zones où les taux de transmission variaient entre 8 et 1000 piqûres d'anophèles infectés par personne et par an. La comparaison de leurs résultats a montré que l'amplitude des taux de mortalité palustre était généralement faible (entre 6 et 10 pour mille annuellement chez les enfants de moins de 5 ans pour neuf études sur onze) et qu'elle était indépendante du niveau de la transmission [14].

\section{Commentaire}

Ces deux approches, à la fois diffé- la complexité des relations hôte/vecteur/parasite dans le paludisme et apportent des réponses apparemment contradictoires. Y a-t-il moyen de les concilier? Une explication semble être apportée par les études effectuées dans les contextes épidémiologiques où la transmission varie selon les saisons; ces études montrent que les fluctuations de la transmission ont des conséquences immédiates sur les taux de morbidité et de mortalité palustres. Dans ces conditions les études d'intervention pourraient simplement mesurer le même type d'effet à court terme. Tandis que les analyses épidémiologiques refléteraient davantage l'équilibre résultant du développement de la prémunition.

La question des conséquences à moyen et long terme d'un programme de lutte antivectorielle se pose en ces termes: un nouvel équilibre entre l'homme et son parasite, limitant dans le temps les améliorations observées, peut-il s'instaurer après quelques années d'intervention? Nous pensons que cette importante question ne peut pas être tranchée au vu des études d'interventions actuellement disponibles. Cela pour trois raisons principales.

1. Les études d'intervention ont duré un ou deux ans seulement, période manifestement trop brève.

2. Dans les contextes de forte prémunition de la population, les conséquences de la réduction de la transmission, si elles sont évaluées sur les seuls jeunes enfants, peuvent conduire à une apparente réduction de la morbidité et de la mortalité palustres. Bien réelle pour les classes d'âge les plus jeunes cette réduction initiale pourrait être progressivement compensée par une morbidité et mortalité qui se maintiendraient après l'enfance (jusqu'à et y compris l'âge adulte), d'autant plus que le nouveau niveau d'exposition sera plus faible; c'est ce que suggèrent toutes les études comparatives entre zones différant par l'intensité de la transmission.

3. Les études d'intervention sont presque toujours accompagnées d'une amélioration de l'information sanitaire et/ou de l'accès aux soins des populations concernées. Cette amélioration peut avoir des conséquences très importantes dans le contexte de l'Afrique rurale: dans un récent essai d'un vaccin antipaludique en Gambie, deux visites domiciliaires hebdomadaires de surveillance médicale ont permis de réduire de plus de $90 \%$ la mortalité des enfants malgré l'absence d'efficacité du vaccin testé [8].

\section{L'enjeu}

Ce débat est d'importance. Son enjeu est la place de la lutte antivectorielle dans la lutte antipaludique en zone de forte et moyenne endémie. Ce débat concerne également le développement de deux axes de recherches qui sont très activement étudiés: la mise au point d'un vaccin antipaludique bloquant la transmission et l'introduction par génie génétique chez les anophèles de gènes de résistance au développement du parasite. L'une et l'autre de ces méthodes ont pour cible la transmission du paludisme et auraient donc en Afrique rurale les mêmes limites que les méthodes déjà disponibles de lutte antivectorielle.

Toute stratégie de lutte antipaludique doit viser à optimaliser un ensemble d'actions relevant: (1) de la prise en charge des malades (éducation sanitaire des populations pour une attitude adaptée face à la maladie; réseau de structures sanitaires; disponibilité en personnels et médicaments; schémas thérapeutiques appropriés...); (2) de la prévention de la maladie, dont la lutte antivectorielle est une des composantes majeures. A l'évidence ces catégories de mesures sont complémentaires et ne sont pas exclusives les unes des autres; mais en pratique, tant la carte des zones de forte endémie et celle des pays les plus pauvres coïncident, tout n'est pas possible en même temps et des choix doivent être faits. La communauté scientifique découvre le débat, est divisée et propose, en retour, trois types de réponses: certains pensent que l'efficacité de la réduction de la transmission a fait ses preuves et qu'il convient en particulier de développer de grands programmes de moustiquaires imprégnées [15, $16]$; d'autres pensent que les argu- 
ments récemment accumulés contre cette efficacité sont déjà suffisamment probants et qu'il faut axer la lutte antipaludique sur l'amélioration de la prise en charge des malades $[17,18]$; enfin, beaucoup s'interrogent sur les arguments avancés et souhaiteraient disposer de davantage de données [19].

\section{Les voies de recherches}

Une prochaine étape pourrait être la réalisation d'une intervention réduisant la transmission dans une vaste zone pendant de nombreuses années $[15,20,21]$. Les observations sur la morbidité palustre au Sénégal suggèrent qu'une intervention d'une dizaine d'années serait nécessaire [17]. Cette intervention soulève de nombreuses questions sur l'ampleur de la réduction de la transmission et/ou de l'exposition du groupe cible qu'il sera possible/souhaitable de maintenir durablement. Citons les principales. Quelles seront les modifications des comportements trophiques des moustiques? Comment prendre en compte ces changements éventuels dans l'évaluation de l'exposition individuelle? L'utilisation à grande échelle des moustiquaires imprégnées ne va-t-elle pas généraliser la résistance des anophèles aux pyréthrinoïdes, déjà observée en Afrique [22] ? Comment prendre en compte une possible évolution de l'acceptabilité de la méthode de lutte par la population? Par ailleurs, d'autres facteurs dont l'importance potentielle n'a pas été prise en compte jusqu'à présent pourraient limiter la signification des résultats obtenus. Comme pour d'autres interventions dans le domaine de la santé, la meilleure méthode pour l'évaluation d'une nouvelle stratégie de contrôle du paludisme est l'essai randomisé en double aveugle. Cela est possible dans le cas d'essais chimiothérapeutiques, chimioprophylaxiques ou vaccinaux, mais difficilement praticables avec d'autres types d'intervention antipaludique; dans le cas de l'utilisation de moustiquaires imprégnées, tant l'utilisateur que l'équipe qui conduit l'essai savent de façon évidente qui relève des groupes protégés ou témoins. Enfin, il existe de nombreuses variables qui sont instables au cours du temps et bien connues pour influencer grandement la morbidité et la mortalité (par exemple l'accès aux médicaments, la chimiosensibilité de $P$. falciparum, les modalités de recours aux soins, la modification de l'état sanitaire global, la survenue d'épidémies, etc.) ; ces variables, dont beaucoup sont difficilement évaluables, pourraient avoir un effet beaucoup plus important que la réduction de la transmission sur l'évolution de la fréquence des accès graves et sur la mortalité palustre dans une classe d'âge donnée.

La réalisation d'études d'intervention de longue durée est délicate et les résultats à en attendre sont probablement limités. En conséquence l'approfondissement de la comparaison de situations où la transmission diffère semble actuellement l'approche la plus réaliste. La comparaison de différentes zones permettra également de mieux comprendre l'importance absolue et relative des nombreux déterminants de la morbidité et de la mortalité palustres. Cette compréhension apparaît de plus en plus comme un préalable indispensable à la définition de stratégies de lutte qui soient efficaces tant à court qu'à long terme dans les zones de forte et moyenne endémie.

Jusqu'à présent les données qui permettent une comparaison de l'incidence des accès simples à différents niveaux de transmission en zone d'endémie stable sont peu nombreuses [17, 23], comme les études permettant une comparaison de l'incidence des accès graves et de la mortalité palustre. Dans la majorité des cas, les méthodes utilisées et les populations étudiées diffèrent tellement que comparer les résultats obtenus est délicat. Ensuite, les problèmes méthodologiques pour la conduite de telles études comparatives sont nombreux, en particulier en ce qui concerne la mesure de la mortalité due au paludisme. D'une façon générale, plus la mortalité palustre est forte, plus la proportion de décès survenant dans des formations sanitaires est faible. Dès lors, attribuer un décès au paludisme ne peut reposer que sur la méthode dite d'autopsie verbale, qui est une interview post-mortem de l'entourage de la personne décédée selon un questionnaire préétabli. La valeur de cette méthode est extrêmement variable selon les causes de décès et reste mal connue dans le cas du paludisme en Afrique. Par ailleurs, les entités cliniques attribuables au paludisme varient en fréquence avec l'âge et le niveau de transmission. Les anémies graves se développent très rapidement chez les nourrissons; elles représentaient autrefois en zone de forte endémie l'essentiel des causes de décès par paludisme [24]. L'autopsie verbale est susceptible de sous-estimer leur fréquence beaucoup plus facilement que dans le cas du paludisme cérébral.

Le concept de contrôle du paludisme, dont l'objectif central est la réduction de la mortalité et de la morbidité occasionnées par cette affection, est né de l'abandon de l'espoir de l'éradication pour le court et le moyen terme. Il soulève de nouveaux défis conceptuels, méthodologiques et opérationnels dont l'importance a longtemps été sous-estimée [25]. Peu de moyens sont consacrés à leur étude et celle-ci ne progresse que lentement. Dans le même temps, les responsables de la lutte antipaludique sont confrontés à une dégradation dramatique de la situation sous l'effet de la progression des chimiorésistances. Face à l'urgence de la situation et au désarroi qu'elle provoque, la tentation est grande, au risque de nouvelles désillusions, de promouvoir l'utilisation à vaste échelle de nouvelles méthodes de lutte qui relèvent encore, en partie, du domaine de la recherche. C'est aussi un élément essentiel à prendre en compte dans le débat actuel

Remerciements

Les auteurs remercient Michel Cot et Pierre Gazin pour la relecture critique du manuscrit.

\section{TIRÉS À PART}

V. Robert. 


\section{RÉFÉRENCES}

1. Carnevale P, Bosseno MF, Zoulani A, Michel R, Molez JF. La dynamique de la transmission du paludisme humain en zone de savane herbeuse et de forêt dégradée des environs nord et sud de Brazaville, R.P. du Congo. Cah ORSTOM Sér Ent Méd Parasitol $1985 ; 23$ : 95-115.

2. Mouchet I, Robert V, Carnevale P, Ravaonjanahary, Coosemans M, Fontenille D, Lochouarn L. Le défi de la lutte contre le paludisme en Afrique tropicale: place et limite de la lutte antivectorielle. Cahiers Santé 1991 ; 1 : 277-88.

3. Payne D, Grab B, Fontaine RE, Hempel $\mathrm{JH}$. Impact of control measures on malaria transmission and general mortality. Bull WHO 1976; 54: 369-77.

4. Barutwanayo M, Coosemans M, Delacollette C, Bisore S, Mpitabakana P, Seruzingo D. La lutte contre les vecteurs du paludisme dans le cadre d'un projet de développement rural au Burundi. Ann Soc Belge Méd Trop 1991; 71 (suppl 1) : 113-25.

5. Robert V, Carnevale P. Influence of deltamethrin treatment of bed nets on malaria transmission in the Kou valley, Burkina Faso. Bull WHO 1991 ; 69 : 735-40.

6. Carnevale P, Robert V, Snow R, Curtis C, Richard A, Boudin C, Pazart LH, Halna JM, Mouchet J. L'impact des moustiquaires imprégnées sur la prévalence et la morbidité liée au paludisme en Afrique subsaharienne. Ann Soc Belge Méd Trop 1991; 71 (suppl 1) : 127-50.

7. Choi HW, Breman JG, Teutsch SM, Liu S, Hightower AW, Sexton JD. The effectiveness of insecticide-impregnated bednets in reducing cases of malaria infection : a metaanalysis of published results. Am J Trop Med Hyg 1995; 52 : 377-82.

8. D’Alessandro U, Olaleye BO, McGuire W, Langerock P, Bennet S, Aikins MK, Thomson MC, Cham MK, Cham BA, Greenwood BM. Mortality and morbidity from malaria in Gambian children after introduction of an impregnated bednet programme. Lancet $1995 ; 345$ : 479-83.

9. Nevill CG, Some ES, Mung'ala VO, Mutemi W, New L, March K, Lengeler C, Snow RW. Insecticide-treated bednets reduce mortality and severe morbidity from malaria among children on the Kenyan coast. Trop Med Int Hlth 1996; 1 : 139-46.

10. Kubaje A, Adjuik M, Williams LA, Lengeler C, Maude GH, Armah GE, Kajihara B, Adiamah JH, Smith PG. Impact of permethrin impregnated bednets on child mortality in Kassena-Nankana district, Ghana: a randomized controlled trial. Trop Med Int Hlth 1996; 1 : 147-54.

11. Trape JF, Quinet MC, Nzingoula S, Senga P, Tchichelle F, Carme B, Candito D, Mayanda H, Zoulani A. Malaria and urbanization in Central Africa: the exemple of lity. Trans $R$ Soc Trop Med Hyg 1987; 81 (suppl 2) : 34-42.

12. Snow RW, Bastos de Azevedo I, Lowe BS, Kabiru EW, Nevill CG, Mwankusye S, Kassiga G, March K, Teuscher. Severe childhood malaria in two areas of markedly different falciparum transmission in East Afric. Acta Tropica 1994; 57: 289-300.

13. Mbogo CNM, Snow RW, Khamala CPM, Kabiru EW, Ouma JH, Githure JI, Marsh K, Beier JC. Relationships between Plasmodium falciparum transmission by vector populations and the incidence of severe disease at nine sites on the Kenya coast. Am J Trop Med Hyg 1995; 52: 201-6.

14. Snow RW, Marsh K. Will reducing Plasmodium falciparum transmission alter malaria mortality among african children? Parasitol Today 1995; 11 : 188-90.

15. Greenwood BM. Malaria transmission and vector control. Parasitol Today 1997; 13 : 90-2.

16. Lengeler C, Smith TA, Schellenberg JA. Focus on the effect of bednets on malaria morbidity and mortality. Parasitol Today 1997; 13 : 123-4.

17. Trape JF, Rogier C. Combating malaria morbidity and mortality by reducing transmission. Parasitol Today 1996; 12: 236-40.

18. Trape JF. Which strategy for malaria control in Africa? Parasitol Today 1997; 13 : $125-6$.

19. D'Alessandro U, Coosemans M. Concerns on long-term efficacy of an insecticide-treted bednet programme on child mortality. Parasitol Today 1997; 13 : 124-5.

20. Snow RM, March K. Will reducing $P$. falciparum transmission alter malaria mortality among African children? Parasitol Today 1995; 11: 188-90.

21. Lengeler C, Snow RW. From efficacy to effectiveness : insecticide-treated bednets in Africa. Bull WHO 1996; 74: 325-32.

22. Elissa N, Mouchet J, Rivière F, Meunier JY, Yao K. Resistance of Anopheles gambiae s.s. to pyrethroids in Côte d'Ivoire. Ann Soc Belge Méd Trop 1993; 73: 291-94.

23. Trape JF, Lefebvre-Zante E, Legros F, Druilhe P, Rogier C, Bouganali H, Salem G. Malaria morbidity among children exposed to low seasonal transmission in Dakar, Senegal and its implications for malaria control in tropical Africa. Am J Trop Med Hyg 1993; 48: 748-56.

24. Kivits M. Pathologie et mortalité de l'enfance indigène au Mayombe. Institut royal colonial belge, Section des Sciences naturelles et médicales, Collection Mémoires 1951; 19 (4) : 1-33.

25. Trape JF, Greenwood B. Approches nouvelles en épidémiologie du paludisme. Ann Institut Pasteur Act 1994 ; 5 : 259-69.
Vincent Robert Jean-François Trape

Institut français de recherche scientifique pour le développement en coopération (Orstom), laboratoire de paludologie, Orstom, BP 1386, Dakar, Sénégal.

E-mail: Vincent.Robert@orstom.sn

\section{Summary}

Controling malaria by reducing transmission ? A controversy

The place of the vector control in the malaria control is currently debated for areas of high and medium endemic malaria (i.e. mainly in rural tropical Africa). The short-term impact of interventions reducing transmission by means of insecticides, such as house spraying or impregnated bednets, shows an important reduction of malarial morbidity and mortality. On the contrary, the comparison of various situations with different levels of malaria transmission suggest that a decrease of transmission would have only consequences in the delay of the mean age at which malaria attacks occur but not in the overall importance of malarial morbidity and mortality. The obvious discrepancy between the observed results reflect different approaches. It reveals above all the importance of the malaria control challenges. 